\title{
An RF Replay System for Narrowband GNSS IF Signals
}

\author{
Staffan Backén, Student Member, IEEE, Dennis M. Akos, Senior Member, IEEE, \\ and Steven G. Wilson, Student Member, IEEE
}

\begin{abstract}
Although GNSS (Global Navigation Satellite Systems) RF (Radio Frequency) signal simulators have long possessed the capability to generate scenarios, they are for example not yet able to model a specific realistic scenario with complex multipath.
\end{abstract}

Software-defined receivers bridge the gap between simulated and real data to the extent that they may offer a replay capability, where a data set is first recorded to disk and later can be processed several times. Unfortunately, the recorded data generally cannot be used by hardware-based GNSS receivers, making receiver-to-receiver comparisons difficult and time consuming.

This paper describes a system capable of replaying recorded IF (intermediate frequency) data into any narrow bandwidth $\mathrm{L} 1$ (the frequency band of the traditional civilian GPS signal) GNSS receiver, including an evaluation of the difference (position, timing, and signal- to-noise ratio) between live and replayed data using a high sensitivity, consumer-grade receiver. The performance of the replayed data set was, with regards to the position and timing, found to match that of the live data.

Manuscript received September 20, 2007; revised April 23,2009; accepted July 28, 2009; revised September 29, 2009. This work was supported in part by the Swedish national graduate school of space technology.

S. Backén and D.M. Akos are with the Department of Electrical Engineering and Computer Science, Luleå University of Technology, Luleå, 97187 Sweden e-mail: staffan@csee.ltu.se

S.G. Wilson is with the Department of Aerospace, Colorado University, Boulder, CO, USA
Index Terms-Global Positioning System, GNSS, RF sampling, GNSS simulator.

\section{INTRODUCTION}

Since the advent of NAVSTAR GPS, test and validation of GNSS receivers have played an important role in development of user equipment. An invaluable tool in this process is GNSS RF simulators (which replace the antenna) that emulate the high frequency signals received by a user at a particular location. The operator has significant control over the scenario as most signal parameters are configurable. Although modern simulators provide features such as multipath (reflection of the incident waves off surrounding surfaces) and signal blockage, it is difficult to accurately model a specific scenario in a complex environment. The reason for this is twofold. First, multipath is modeled inside the simulator using separate hardware channels and the number of channels is limited. Secondly, the required information to accurately model a specific environment, using for example raytracing as in Jülg [1] or Weiss et al. [2], is generally not available.

With the advent of software receivers, the capability to record IF (intermediate frequency, a lower frequency replica of the RF GNSS signals) data sets provides developers and researchers with the option of processing 
real, recorded data an infinite number of times. Unfortunately, this method is not available for users of hardware-based receivers, where the options for performance evaluation are either field tests (time consuming) or simulator scenarios. See [3] for an overview of both methods applied to automotive testing. This paper describes a replay system where a standard GNSS front-end is used to digitize the signal and a data bridge is used to store the samples to disk. Next, the data bridge is run backwards to feed the samples into the playback instrument consisting of a DAC (digital-to-analog converter), filters, and a mixer to shift the signal up to the nominal GPS L1 carrier frequency $(1575.42 \mathrm{MHz})$. After the signal has been attenuated, it can be fed into any narrow band GNSS receiver.

For readers new to GNSS, Misra and Enge [4] is an excellent overview, while Kaplan and Hegarty [5] provide a more in-depth treatment of various aspect of the topic. The replayer was proposed by Brown et al. [6], and Pósfay et al. investigated replay functionality at the IF level with the intent of mixing to RF [7]. The functionality of the replay system is similar to a simulator as scenarios can be played back indefinitely through any GNSS receiver. This have several advantages; for example, a data set can be replayed several times into a specific receiver to provide statistical estimates of performance, data sets recorded in environments that are hard to recreate can be reused at no additional expense and new receiver models can be verified against a data set recorded in the past.

The replay system can record data sets in challenging environments - under conditions (urban canyons, indoors) that can be difficult, or at least time consuming, to model realistically in a simulator. Given the importance, and corresponding effort, directed at addressing, for example, indoor navigation, a replay system capable of offering insight into the signal environment could be very useful. Contrary to a simulator, control of the scenarios are limited to the environment in which the data set was recorded and any truth reference would require an advanced GPS/INS system with differential post processing to be used in recording - and only be as accurate as such a system would allow. To summarize, an RF replay system would be a very capable complement to traditional simulators and help advance GNSS research.

The paper is organized as follows: the two major modes of operation, recording and playback, are explained in section II and III. Important design considerations such as filter bandwidths and noise figure calculations are covered in section IV. In $\mathrm{V}$, the performance of a replayed data set is compared to its live counterpart with regards to position, timing, and SNR (signal-tonoise ratio). Finally, conclusions are stated in section VI.

\section{RECORDING SYSTEM}

The replay system has two modes of operation: recording and playback. During recording, the high frequency signal is conditioned (filtered and translated in frequency to a lower rate), sampled, and stored to disk.

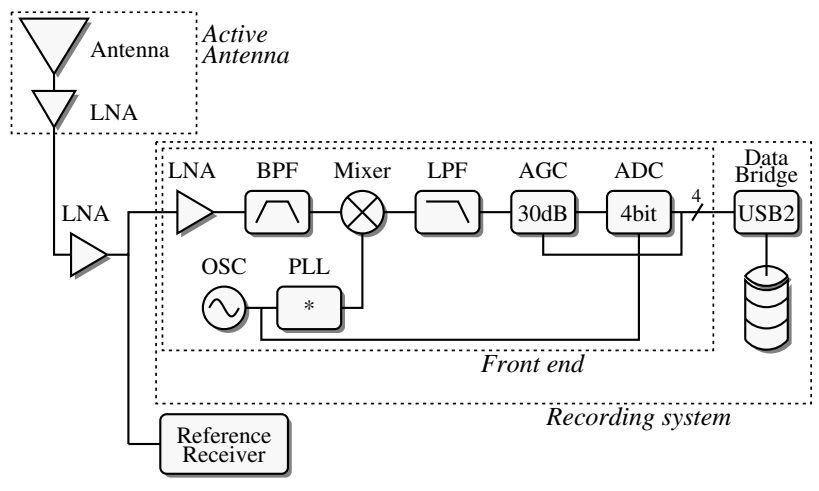

Fig. 1. Recording of IF data 
Figure 1 shows the setup of the replay system used to record data. First, a roof-mounted survey-grade antenna with a built-in amplifier receives the signal around the nominal GPS L1 center frequency, in this case 1575.42 MHz. After amplification, filtering, down mixing, and quantization in the front-end, the resulting IF signal is recorded to disk. Also, in order to validate the performance of the replay system, the RF signal is simultaneously processed by a consumer-grade, narrow bandwidth receiver in real time. Note that the oscillator can be synchronized to an external frequency reference by means of a standardized interface.

\section{A. Front-end}

As part of figure 1, a sample GNSS front-end is shown where the RF signal is first amplified and band pass filtered. The amplification is performed by low-noise amplifiers (LNA), increasing the power level of the noise from a nominal level of $800 \mathrm{nV}$ (when received by the antenna) to around $1 \mathrm{~V}$ (at the input of the ADC). After mixing (multiplication with a sinusoid with a frequency close to $1575.42 \mathrm{MHz}$ ), the signal is centered around an IF suitable for sampling. Then, a low pass filter is used to remove the spectral image and minimize aliasing losses due to violation of the Nyquist-Shannon sampling theorem. An AGC (automatic gain control) adjusts the signal power for optimal quantization prior to sampling. Borre et al. covers the design of a low-cost front-end in [8].

The bandwidth of the front-end used for this experiment is approximately $2.5 \mathrm{MHz}$, and the IF and sampling frequencies are $4.1304 \mathrm{MHz}$ and $16.3676 \mathrm{MHz}$ respectively.

\section{B. Data bridge}

The role of the data bridge is to facilitate storage of digital samples. In this case, the data bridge provides an interface between the high rate data from the front-end and a host PC.

The protocol for this implementation is High Speed USB v2.0, which implies that the maximum data transfer rate is $480 \mathrm{Mbits} / \mathrm{s}$, although achievable rates are typically quite lower [9]. Most narrow band front-ends have a sampling frequency between 4 and $20 \mathrm{MHz}$ and a resolution of $2-4$ bits, thus the transfer bandwidth is sufficient for narrow band receivers. However, it is not adequate for high bandwidth receivers (such as survey grade), nor is it suitable for interference mitigation receivers requiring high (more than 8 bits) dynamic resolution. The ADC in the recording front-end used in this experiment has a resolution of 4 bits.

The performance of the storage system is crucial for this kind of setup. Modern individual hard drives for desktop computers are generally capable of transferring about $60 \mathrm{MB} / \mathrm{s}$ continuously; however, high speed reading of data is the more difficult problem than continuously writing at the same rate. The reason is that when the hard drive is temporarily unavailable during writing, the memory buffer that temporarily stores the data in RAM can easily be extended (write-behind) to allow for a short outtake, whereas during reading the buffer needs to be extended before (read-ahead) the outtake. This problem could be solved in the application software with the use of a large data buffer when reading, or by using solid state disks with a significantly lower latency. It was however considered out of scope for this proof of concept system. In addition, the host PC chip set design/stability can have a major impact on the resulting performance of such an application.

\section{Playback System}

The second mode is playback, where the recorded signal is converted back to a high frequency signal 
and connected to a GNSS receiver. Figure 2 shows the

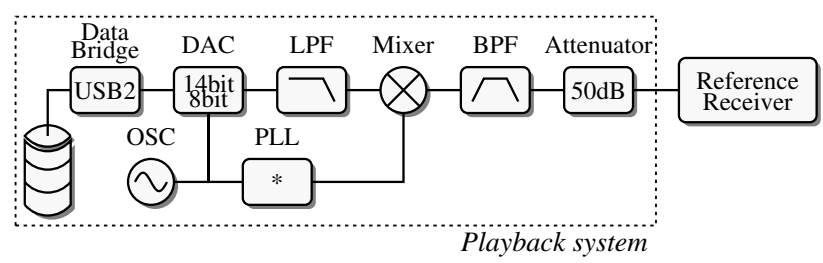

Fig. 2. Playback of RF data

functionality of the playback component. Essentially, it works as an inverted front-end. The first step is to convert the digital samples to analog in the DAC (for the system discussed in this paper an 8 bit DAC was used). Next, a low pass reconstruction filter removes high frequency components from the conversion process. In the mixing stage, the signal is moved up to the correct carrier frequency (in this case, $1575.42 \mathrm{MHz}$ ) and band pass filtered to remove the image signal. After significant attenuation to emulate the power/noise output characteristics of an active antenna, the signal can be processed by any L1 narrow band GNSS receiver.

As an example, consider a signal received by an active antenna. The received noise power is nominally $-111 \mathrm{dBm}$ (assuming an ambient temperature of $273 \mathrm{~K}$ ) in a $2 \mathrm{MHz}$ bandwidth. Active GNSS antennas have built-in amplifiers with a gain of about $25 \mathrm{~dB}$, and GNSS receivers are thus designed to receive signals with a power around $-86 \mathrm{dBm}$ with some upper and lower margin provided by the AGC. If the ADC in the front-end used to record our signal (figure 1) has a desired input voltage around $1 \mathrm{~V}$, the signal requires an additional amplification of about $75 \mathrm{~dB}$. Returning to the replay system, and noting that the DAC provide a maximum output level of $1 \mathrm{~V}$ as well, it is evident that the output power of the replay system is too high for the receiver. Thus, the signal requires attenuation after the replay system but before the input to the receiver under test. Due to differences between our approximate estimates above and the actual specifications of our system, the suitable attenuation was determined to be $50 \mathrm{~dB}$ (as shown in figure 2) as opposed to $75 \mathrm{~dB}$. This was verified using the reported AGC level of the receiver under test.

The stability of the replay system is, after a few modifications, acceptable for research applications where rare failures are non-critical. The 30 minute data set used for the experiment outlined in this paper failed once in 5 tests, giving some insight of the failure rate. It is reasonable to believe that the failures are due to the read-ahead issue mentioned previously. Choosing a motherboard with a well-renowned USB2 chipset is recommended (temperature issues were identified with several mother board vendors). The target platform is Linux which proved to be a wise choice for several reasons (stability, process optimization, and available driver code).

Using an ideal recording and playback system (that introduces no additional noise, no aliasing, and has perfect oscillator); the receiver under test would not be able to distinguish whether it is connected to an antenna or our replay system after a cold start (a cold start means that the receiver has no a priori information). If the only non-ideal effect is a small frequency offset, the receiver cannot differentiate between that and the impact from its internal clock having the same offset. This may at first be considered a strong statement, but it is an effect of fundamental information theory. Obviously, physical and practical constraints limit the performance that can be achieved.

As in the recording front-end, the replay system also offers the option of interfacing different frequency sources using a standardized interface. 


\section{DESIGN CONSIDERATIONS}

In this section, several of the design choices for the replay part of the system will be explained, together with a general explanation of their impact on the complete system.

\section{A. Digital bandpass and requantization}

Generally, the frequency spectrum of GNSS front-ends tends to have a non negligible DC component that has a minimal impact on signal processing. However, in a replay system, this may limit the effective dynamic range of the DAC. In order to minimize this, the recorded 4 bit data was filtered with a high order band pass filter centered around the IF with a bandwidth of $4 \mathrm{MHz}$. The filter type was FIR (finite impulse response) with floating filter constants. In order to fully use all 8 bits of the DAC, the filtered signal was amplified with a constant so that the full dynamic range would be used after quantization to signed 8 bit integers. Not much is to be gained by using a resampling bandwidth larger than either the recording front-end or the target receiver, thus the choice of a $4 \mathrm{MHz}$ bandwidth. It is important to note the difference between the signal received by an antenna/produced by a simulator and the signal generated by a replay system. The former two are essentially not band-limited with regards to the $2 \mathrm{MHz}$ wide C/A code signal (antennas at L1 typically have a fairly large bandwidth). The bandwidth of the signal reconstructed in the replay system is limited by all filtering components but in general, as long as it is wider than the receiver under test, the filtering has a minimal effect on performance.

\section{B. Low pass filter}

The DAC unfortunately produces slight aliasing components, so a $6 \mathrm{MHz}$ low pass filter was inserted after the DAC to make sure the input to the mixer was band limited. The low pass filter is the component directly following the DAC in figure 2.

\section{Band pass filter}

The mixer multiplies the input signal (with frequency $f_{0}$ ) with a sinusoid of a known frequency $f_{m i x}$, effectively translating the frequency of the signal to both $f_{0}+f_{m i x}$ and $f_{0}-f_{m i x}$. Only the latter is of interest, thus a band pass filter around the carrier frequency of interest is recommended. In figure 2, the band pass filter follows the mixer.

\section{Noise figure}

Friis formula for $N$ successive stages of a receiver says that the total noise figure $F_{t o t}$ can be expressed as

$$
F_{t o t}=1+\sum_{n=1}^{N} \frac{F_{n}-1}{\prod_{k=0}^{n-1} G_{k}} ; G_{0}=1
$$

where $G_{n}$ is the power gain of the $n$th stage. Both $F$ and $G$ are expressed as ratios.

As the noise figures are not known for all components (either only specified within a range or reasonable estimates), a best/worst case has been computed as shown in table I. The quoted noise figure for all electrical components (antenna, cable etc.) is assembled from data sheets for either actual parts or similar models. The noise figures for the digital components (filter and requantization) are reasonable estimates. Based on the computation the expected signal-to-noise ratio degradation will fall between 0 and $2.5 \mathrm{~dB}$. This degradation is an estimate of the additional noise caused by using a replay system instead of connecting the receiver directly to the antenna. For this to asymptotically approach $0 \mathrm{~dB}$ the recording/playback quantization should have high dynamic range compared to the receiver, and the antenna gain should be high. 
THIS WORK HAS BEEN ACCEPTED BY THE IEEE AND IS PENDING PUBLICATION. COPYRIGHT MAY BE TRANSFERRED WITHOUT NOTICE, AFTER WHICH THIS VERSION MAY NO LONGER BE ACCESSIBLE.6

TABLE I

NOISE FIGURES, COMPONENTS

\begin{tabular}{lll} 
Component & Noise figure & Gain \\
& best/worst $(\mathrm{dB})$ & best/worst $(\mathrm{dB})$ \\
\hline Antenna & 2.5 & 27.0 \\
Cable & $3.36 / 5.58$ & $-3.36 /-5.58$ \\
Amplifier & $1.55 / 1.90$ & 20.0 \\
Front-end & $2.35 / 4.67$ & $35.2 / 29.0$ \\
Digital filter & 0.0 & 0.0 \\
Requantization & $0.1 / 1.0$ & $3.0 / 0.0$ \\
DAC & $20 / 40$ & $10 / 0$ \\
Low pass filter & $0.3 / 2.0$ & $-0.3 /-2.0$ \\
Mixer & $8.0 / 9.0$ & $20.0 / 10.0$ \\
Band pass filter & $0.5 / 5.0$ & $-0.5 /-5.0$ \\
Attenuator & $50 / 70$ & $-50 /-70$ \\
\hline
\end{tabular}

\section{E. Clock frequency and phase noise considerations}

There are two clock-related issues to consider when designing GNSS hardware; frequency error and jitter. The frequency error issue for this design is best explained with an example, where the receiver under test has a front-end similar to figure 1. The front-end used for this experiment have a PLL (phase-locked loop) that multiplies the sampling frequency by a factor of 96 to generate the LO (Local Oscillator, a sinusoid used to mix the RF signal down to IF).

Further, the TCXO (temperature compensated crystal oscillator) has a maximum error of $1 \mathrm{ppm}(16 \mathrm{~Hz})$. The PLL will multiply this frequency error by a factor of 96, yielding a maximum frequency error at $\mathrm{L} 1$ of about $1.6 \mathrm{kHz}$. Provided the search space has been limited to the maximum Doppler of received signals (on the order of $4 \mathrm{kHz}$ ) plus this maximum frequency error, a total search space of $\pm 6 \mathrm{kHz}$ is required to acquire all signals. The sum of the frequency error at L1 due to the recording front-end (in figure 1) and the replay instrument (left part of figure 2) must be small with regards to $1.6 \mathrm{kHz}$, so as not to push the resulting carrier frequencies outside of the $\pm 6 \mathrm{kHz}$ band. Use of a rubidium/cesium or OCXO (oven controlled crystal oscillator) is recommended to mitigate this effect.

Jitter, and its frequency domain equivalent phase noise, is a stochastic process such that a sinusoid (or a sampling clock) has a random frequency variation over time. In the case of the ADC in figure 1, the difference between successive sampling instances will not be constant. Also, the LO signal will have a similar effect on the mixer and thus affect the IF signal. In our complete system, the received signal is first mixed down and sampled (recording front-end), converted to analog and mixed up (replayer), and finally mixed down and sampled (receiver under test). However, Nadig and Raghipathy [10] showed that the LO phase noise dominates over the sampling clock jitter, and also that the SNR degradation is small for reasonable jitter RMS values. Based on their conclusions, we have no reason to believe that the phase noise of the replay system, given an oscillator with frequency drift and phase noise characteristics consistent with that used in survey grade GPS receivers, will impact the $C / N_{0}$ more than $1 \mathrm{~dB}$ based on conducted testing.

\section{F. Interference}

As mentioned previously, the low number of bits (4) in the recording system precludes its use in environments with high levels of interference. In general however, a replay system can be a valuable instrument when evaluating the impact of interference.

Note that in the system described in this paper, the signal power at the input of the ADC of the first stage (recording) will be constant over time. This means that the AGC of the second stage (playback) and third stage 
(receiver under test) will also be constant. This behavior may not be desired; rather the $\mathrm{AGC}$ of the receiver under test may be of interest.

Let us consider a recording system capable of storing an accurate IF replica of the frequency band of interest when interference is present. The system should ideally not have an AGC but rather a manual control of the gain. The gain should be set such that the ADC uses all bits when the maximum level of interference is present. Further, it should have a dynamic resolution such that a sufficient number of bits are used when the interference is not present.

Similarly, the playback system should also not have an AGC and use as many bits as the recording system. This will provide the receiver under test with the most realistic signal possible. This scheme may be difficult to implement since the maximum interference level can be hard to quantify.

To get around this, GNSS signals could be recorded in an ambient environment where no interference is present using a low dynamic resolution. Then, digital interference signals can be added to the data set, and the playback system suggested above can be calibrated to allow for realistic reproduction of arbitrary interference.

\section{Performance Validation}

In order to validate the performance of the replay system, a 30 minute dataset was recorded using the setup in figure 1. Several key messages from a high sensitivity receiver were recorded simultaneously as the IF data was continuously stored to disk. After filtering and requantization, the IF data was played back into the same receiver used during the recording and the performance was evaluated.

\section{A. Live performance}

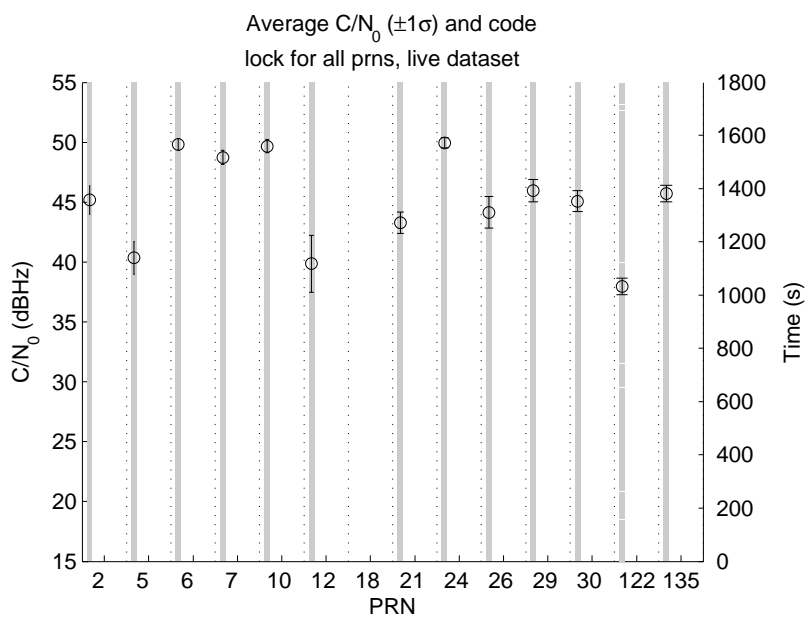

Fig. 3. Code lock and average $C / N_{0}$, live data

Figure 3 shows the code lock (the bar is grey at a time when the receiver maintains lock) and average $C / N_{0}$ for the live data set. PRN (pseudo random noise) in the context of GNSS defines a specific $\operatorname{code}^{1}$ that is transmitted from the satellite. For GPS, each satellite has a unique code and they are numbered from 1 to 32 ( $\mathrm{x}-$ axis). SBAS (space based augmentation systems) such as WAAS (wide area augmentation system) use higher numbers. PRN 122 and PRN 135 are codes transmitted from two WAAS satellite visible during the experiment. As the received SNR could be considered static for shorter static data sets, the mean (center of the black circle) and standard deviation (error bars) of the $C / N_{0}$ have been computed.

As the receiver had ample time to achieve code lock and the signals were sufficiently strong, there are no significant outages in this data set. The highest $C / N_{0}$ level is slightly above $51 \mathrm{dBHz}$, which is expected.

\footnotetext{
${ }^{1}$ A specific PRN number may also define a specific satellite, the
} terms are sometimes used interchangeably but the meaning is generally clear from the context. 


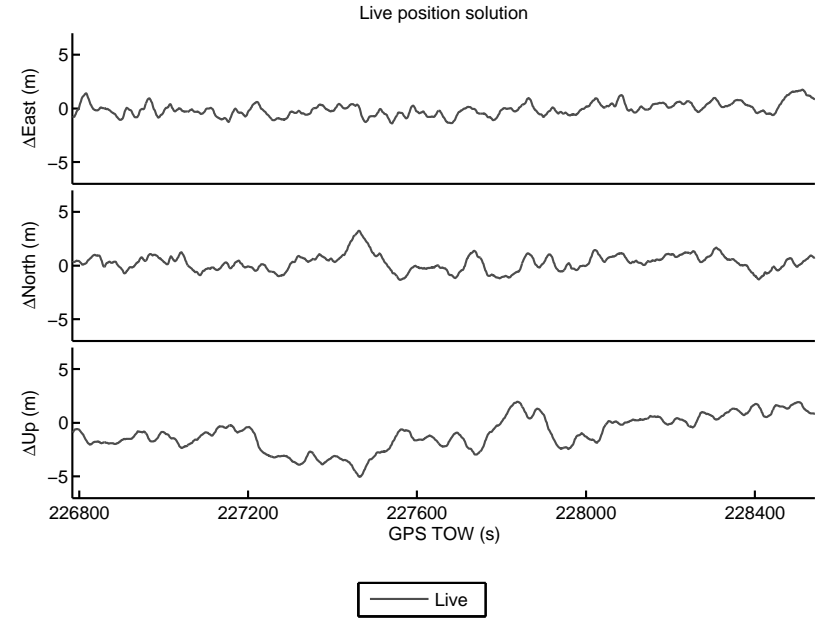

Fig. 4. Position, live data

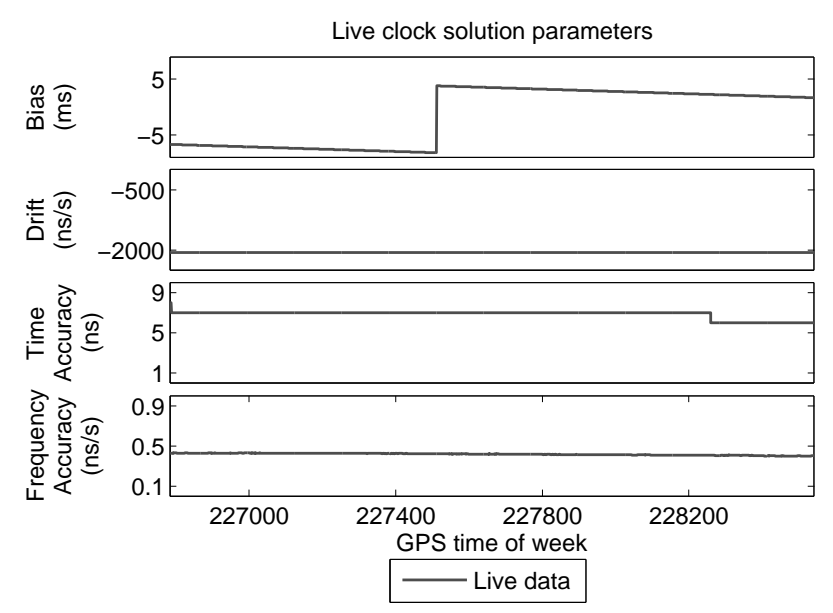

Fig. 5. Clock, live data

The position solution during the live recording is shown in figure 4 and the clock performance is shown in figure 5. The variation of the position solution is within expected limits for a narrow band consumer-grade receiver using WAAS with a full sky view, and also consistent with the accuracy reported by the manufacturer.

Regarding the clock performance (shown in figure 5), first let us clarify certain key parameters. Clock bias is the receiver's estimate of the difference between the receiver local time (derived from the local oscillator) and the time obtained from the position solution. It is common in GNSS receiver design to let this bias increase until it reaches a certain threshold and then reset it. Clock drift is the derivative of the clock bias, and is related to the frequency error in the receiver. The receiver used in this experiment further provides estimates of time and frequency accuracy.

It is evident that the receiver tested here internally allows the clock bias to drift within certain limits as shown in the topmost subplot. Further, the oscillator frequency offset (shown in the second subplot from the top) is fairly steady at about $-2 \mathrm{ppm}$, which is well within the bounds of a standard TCXO.

\section{B. Digital filtering and requantization}

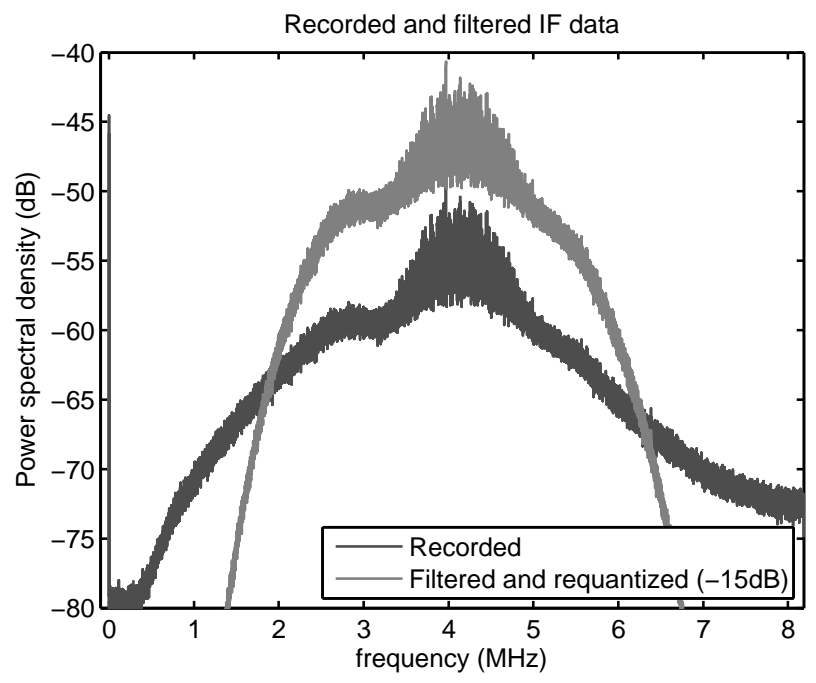

Fig. 6. Spectrum of original and filtered signal, the filtered signal offset slightly to simplify comparison

Figure 6 shows the power spectral density of both the recorded 4 bit data set and the filtered, requantized 8 bit data set used for playback. Please note that the filtered spectrum, with higher dynamic range and thus 
higher spectral density, is offset $-15 \mathrm{~dB}$ for convenient comparison with the original spectrum.

\section{Replay performance}

In order to study the impact of playback resolution and clock drift, the recorded 30 minute data set was played back using three different configurations. Table

TABLE II

DATA SET CONFIGURATIONS

\begin{tabular}{llll}
$\#$ & type & resolution & Reference Oscillator \\
\hline 1 & live & - & - \\
2 & replayed & 8 bits & Rubidium \\
4 & replayed & 8 bits & TCXO \\
3 & replayed & 1 bit & Rubidium
\end{tabular}

II shows the live and 3 different replayed data sets and their respective configurations. During the recording of the data set, the front-end was synchronized to a rubidium frequency standard to minimize clock drift. The same oscillator was used as a reference during the replay of the second and fourth replay experiments, but for the third set, the built in TCXO in the arbitrary waveform generator was used. As the replay system will incorporate drifts and biases from two additional clocks (one in the sampling front-end and a second in the replayer), thus total clock bias and drift will accumulate and appear in the receiver clock solution. It is advisable to use a high quality frequency reference in the recording and replay systems to minimize this effect.

Figure 7 shows the code lock and average $C / N_{0}$ for all four data sets (left to right bar for each PRN) as specified in table II. No PRNs were assigned to specific channels for any of the data sets, as evident from the plot (the live dataset locks on to PRN 122, while the replayed data sets all lock on PRN 18). Further, the loss of lock

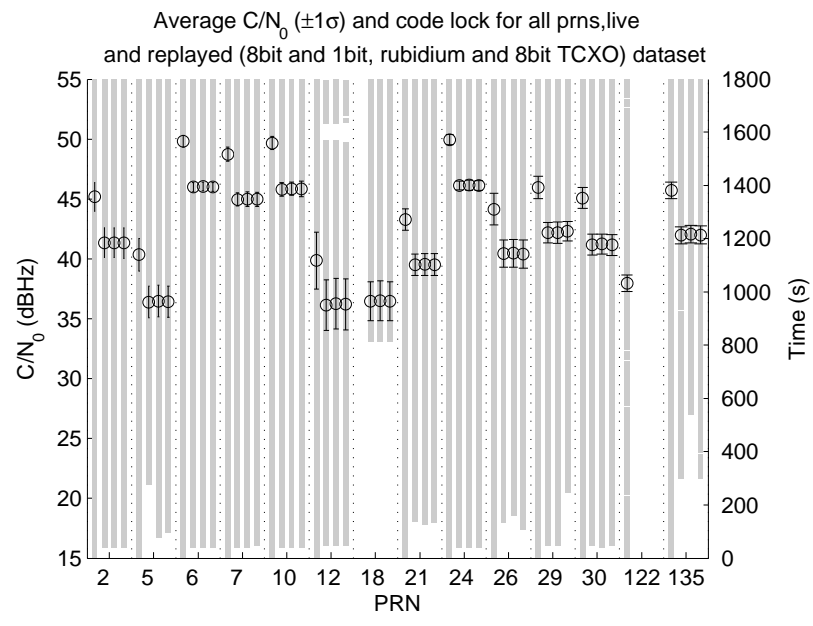

Fig. 7. Code lock and average $C / N_{0}$, all data sets

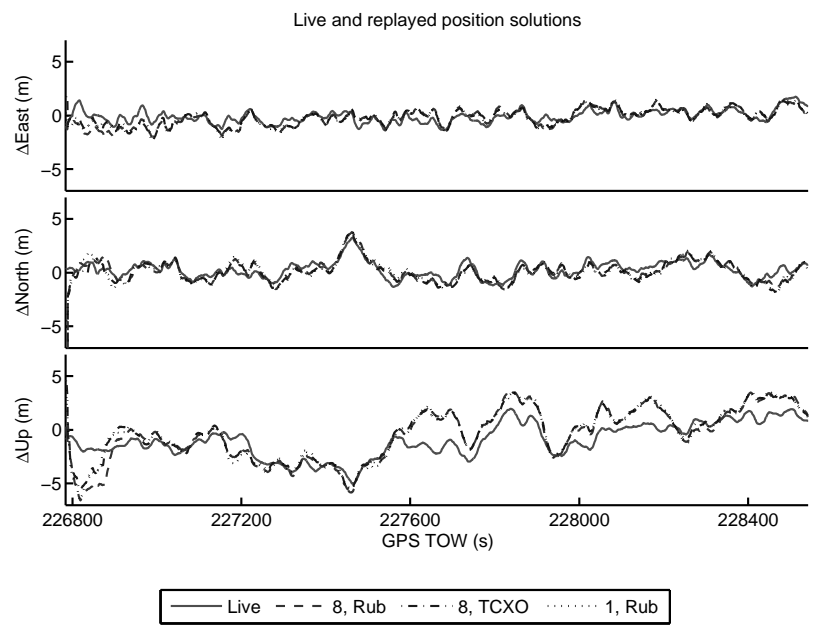

Fig. 8. Position, all data sets

of PRN 12 for all three replayed sets towards the end (the gray bars turn white, slightly before 1600 seconds) is most likely due to a low signal-to-noise ratio.

The position solution of all four data sets is shown in figure 8. All data sets are very similar, and the difference is mainly between the live and the replayed data sets (especially for the vertical position). However, it is a reasonable assumption that the majority of the difference can be attributed to: A. the geometry of the 
satellite signals (the receiver under test acquired satellites in a different order/time during the test as a result of its acquisition logic); and B. the approximate $4 \mathrm{~dB}$ difference in $C / N_{0}$. Little can be done regarding the acquisition logic implemented within the receiver. The approximate $4 \mathrm{~dB}$ difference in $C / N_{0}$ is a result of the added noise in the replay system as well as quantization losses. Additional dynamic range ( $8+$ bits) in the signal recording can help negate this loss.

TABLE III

STANDARD DEVIATION OF POSITION

\begin{tabular}{lllll} 
Direction & Live & 8 bit Rub. & 8 bit TCXO & 1 bit Rub. \\
\hline East & 0.60 & 0.79 & 0.75 & 0.76 \\
North & 0.77 & 0.93 & 0.94 & 0.95 \\
Up & 1.58 & 2.45 & 2.28 & 2.37
\end{tabular}

Table III shows the estimated standard deviation of the east, north, and up components for the four data sets. It was estimated during a time span common to all four data sets. The initial 10 seconds after the receiver reported valid position fixes for all four data sets was excised to mitigate the effect of outliers on the statistical estimates.

As explained in section IV-E, the frequency error due to the recording front-end and the replay system should be small compared to the frequency error due to the receiver under test.

In figure 9, the expected effect of reference frequency error is illustrated. Two of the three replayed data sets use a rubidium frequency standard and hence have a drift that is very close to the live data set $(-2000 \mathrm{~ns} / \mathrm{s}$, or $-2 \mathrm{ppm})$. The replayed set using a TCXO as a reference introduces an additional drift of $1500 \mathrm{~ns} / \mathrm{s}$. The two lower subplots (stability estimates of the clock solution) show acceptable differences between the live and replayed data

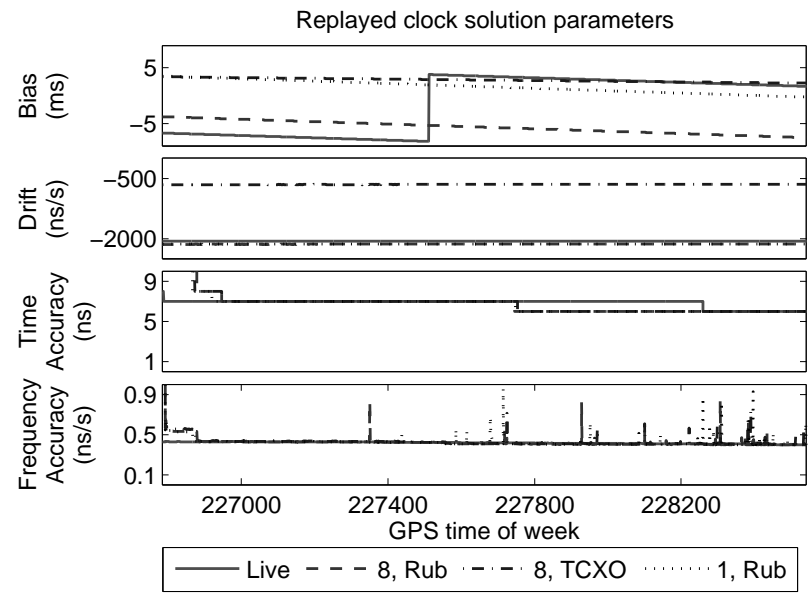

Fig. 9. Clock, all data sets

sets.

\section{Summary AND CONClusions}

A prototype system for a replayer of narrow band GNSS signals has been presented and its performance has been evaluated with regards to position, clock accuracy, and $C / N_{0}$.

The described replay system provides a method by which IF data can be replayed through any narrow band GNSS receiver. The data source can either be actual recorded data (as tested in this paper) or simulated.

The concept may prove to be a very valuable tool in many areas of satellite navigation research, offering a complement to RF signal simulators. A simulator provides control of the scenario and provides a reference position. However, while complex multipath can be generated in high-end simulators, a realistic model would require a detailed map of a certain area and ray-tracing techniques. The system outlined in this paper natively provides this functionality.

Automotive and pedestrian use case testing in urban canyons (where high unpredictable levels of multipath are a problem) is just one example where replay systems 
can help advance the understanding of GNSS receivers. More exotic areas where the replay concept can aid research include, for example, pre-correlation beam forming for antenna array research and interference mitigation algorithm development.

The implementation described and tested in this paper is limited both in bandwidth and dynamic range (the restrictions follow directly from the specifications of the front-end, DAC and data bridge). However, the concept in general is not bound by those constraints and can be extended given available hardware.

\section{REFERENCES}

[1] T. Jülg, "GNSS accuracies under multipath propagation conditions," Proc. IEEE 5th Int. Symp. on Spread Spectrum Techniques and Applications, vol. 3, pp. 709-713, September 1998.

[2] J. P. Weiss, P. Axelrad, and S. Anderson, "A GNSS code multipath model for semi-urban, aircraft, and ship environments," ION Journal of Navigation, vol. 54, no. 4, pp. 293-307, 2007.

[3] D. Aloi, M. Alsliety, and D. Akos, "A methodology for the evaluation of a GPS receiver performance in telematics applications," IEEE Trans. Instrum. Meas., vol. 56, no. 1, pp. 11-24, Feb 2006.

[4] P. Misra and P. Enge, Global Positioning System - Signals, Measurements and Performance, 2nd ed. John Wiley and sons, 2006.

[5] E. Kaplan and C. Hegarty, Understanding GPS, Principles and Applications. Artech House, 2006.

[6] A. Brown, N. Gerein, and K. Taylor, "Modeling and simulation of GPS using software signal generation and digital signal reconstruction," Proc. ION NTM, 2000.

[7] A. Pósfay, T. Pany, and B. Eissfeller, "First results of a GNSS signal generator using a PC and a digital-to-analog converter," Proc. ION GNSS, 2005.

[8] K. Borre, D. Akos, N. Bertelsen, P. Rinder, and S. Jensen, A Software-Defined GPS and Galileo Receiver. Birkhuser, 2007.

[9] M. Junered, "Enabling hardware technology for GNSS software radio research," Licentiate thesis, Luleå University of Technology, 2007.

[10] S. Nadig and A. Raghupathy, "Performance of a GPS receiver in the presence of clock jitter," Proc. NTM GNSS, 2006.

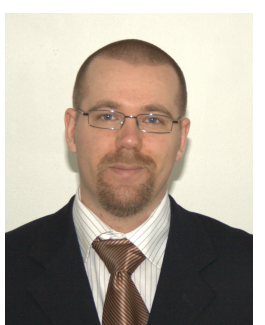

Staffan Backén is a doctoral student at Luleå University of Technology. His research is directed towards antenna array processing of GNSS signals, software receivers and RF electronics. Mr. Backén obtained an M.S. degree in electrical engineering from Luleå University of Technology in 2004.

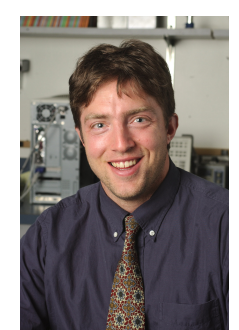

Dennis M. Akos completed the Ph.D. degree in electrical engineering at Ohio University within the Avionics Engineering Center. His research interests include: GNSS, SDR, applied/digital signal processing, and RF design. Currently he is an assistant professor with the Aerospace Engineering Science Department at University of Colorado at Boulder and holds a visiting professor appointment at Lule University of Technology and a consulting professor appointment with Stanford University.

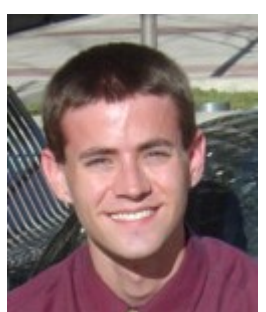

Steven G. Wilson received his M.S. and B.S. degrees in Aerospace Engineering Sciences from the University of Colorado at Boulder in 2009, with a research focus on GPS applications. Currently he works in the Navigation Systems Division of Northop Grumman. 